\title{
Long-term results of biventricular repair after initial Giessen hybrid approach for hypoplastic left heart variants
}

Can Yerebakan, MD, Josephine Murray, MS, Klaus Valeske, MD, Josef Thul, MD, Hatem Elmontaser, MD, Matthias Mueller, MD, Valesco Mann, MD, Stefan Ostermayer, MD, Heiner Latus, MD, Christian Apitz, MD, Dietmar Schranz, MD, and Hakan Akintuerk, MD

Objective: This article presents the long-term results of our patients with a diagnosis of hypoplastic left heart syndrome (HLHS), hypoplastic left heart complex (HLHC), and variants who received a biventricular repair following hybrid stage I with ductal stenting and bilateral pulmonary artery banding.

Methods: Between June 1998 and June 2013, a total of 154 patients with hypoplastic left heart structures underwent a hybrid stage I procedure. Forty patients were definitely treated by creating a biventricular circulation. Median age and body weight of patients before hybrid stage I were 8.5 days (2-40) and $3.0 \mathrm{~kg}$ (1.6-3.8), respectively. The diagnoses were HLHS with mitral and aortic stenosis $(\mathrm{n}=7)$, HLHC $(\mathrm{n}=15)$, HLHC with interrupted aortic arch $(n=9)$, critical aortic stenosis with hypoplastic aortic arch $(n=4)$, imbalanced atrioventricular septal defect with hypoplastic aortic $\operatorname{arch}(\mathrm{n}=2)$, double-outlet right ventricle with hypoplastic aortic $\operatorname{arch}(n=2)$, and d-transposition of the great arteries with interrupted aortic arch $(n=1)$. Median age at the time of biventricular correction was 6.7 months (1.6-13.8). The patients were treated with direct biventricular correction, including repair of intracardiac defects $(n=32)$, Norwood/Rastelli or Yasui $(n=4)$, arterial switch $(\mathrm{n}=2)$, Rastelli $(\mathrm{n}=1)$, and Ross-Konno $(\mathrm{n}=1)$ operations with ascending aortic/aortic arch reconstruction.

Results: All patients survived hybrid stage I. Median survival after biventricular correction is 7.9 years (0.914.9). Overall mortality was $10 \%$ (4 patients) at 4 weeks, 5 weeks, 6 weeks, and 4 months after biventricular correction, respectively. One patient had to be switched to univentricular circulation and another patient underwent orthotopic heart transplantation 3 and 4 months after biventricular correction, respectively.

Conclusions: The Giessen hybrid approach is an alternative to the conventional strategy to treat neonates with HLHS, HLHC, and variants. Biventricular repair after hybrid stage I is feasible and can be performed with satisfactory long-term survival. (J Thorac Cardiovasc Surg 2015;149:1112-22)

See related commentary on pages 1122-3.

$\mathcal{B}$ Supplemental material is available online.

The management of a "small left heart" continues to be a challenging area in congenital heart disease. Numerous

\footnotetext{
From the Pediatric Heart Center Giessen, Justus-Liebig University, Giessen, Germany.

Disclosures: Matthias Mueller reports lecture fees from Orion Pharma GmbH, lindehealthcare, and Sorin Deutschland GmbH. Valesco Mann reports lecture fees from Orion Pharma. The other authors have nothing to disclose with regard to commercial support.

Read at the 94th Annual Meeting of The American Association for Thoracic Surgery, Toronto, Ontario, Canada, April 26-30, 2014.

Received for publication April 24, 2014; revisions received Sept 4, 2014; accepted for publication Sept 7, 2014; available ahead of print Oct, 20, 2014

Address for reprints: Hakan Akintuerk, MD, Pediatric Heart Center Giessen, Department of Congenital Heart Surgery, Justus-Liebig University, Feulgenstrasse 10-12, 35385 Giessen, Germany (E-mail: can.yerebakan@chiru.med.uni-giessen.de).

$0022-5223 / \$ 36.00$

Copyright (C) 2015 by The American Association for Thoracic Surgery

http://dx.doi.org/10.1016/j.jtcvs.2014.09.028
}

studies have attempted to determine suitable candidates for a biventricular repair in the newborn period. ${ }^{1-3}$

Patients with a small left heart belong to a spectrum of a wide variety of possible combinations of hypoplastic/obstructive left-sided lesions. For certain cases with, for example, almost absent left ventricular cavity, the therapeutic route of a univentricular palliation is well defined. In recent years, early prenatal diagnosis, immediate postnatal recovery with prostaglandins, and novel strategies for extracorporeal circulation in conjunction with refined techniques for surgical palliation or hybrid strategy and intensive care management have resulted in remarkable improvements in the outcome for patients. ${ }^{4-6}$

A demanding controversy, however, exists in the management of the subset of patients with borderline small left heart or, for example, a hypoplastic left heart complex (HLHC). HLHC comprises small/obstructive left-sided lesions, ductal-dependent circulation of the lower body, and antegrade flow to the ascending aorta (exception: aortic atresia and ventricular septal defect with 2 developed ventricles). ${ }^{7}$ For this group, the treatment strategy with regard to universus biventricular pathway or heart transplantation ideally ought to be correctly determined in the newborn period to achieve a favorable outcome. Well-known interstage and long-term challenges of a univentricular circulation 


\section{Abbreviations and Acronyms \\ $\mathrm{BVC}=$ biventricular correction \\ HLHC $=$ hypoplastic left heart complex \\ HLHS $=$ hypoplastic left heart syndrome \\ VSD $=$ ventricular septal defect}

following a multistage palliative approach ending with Fontan completion makes a biventricular circulation preferable. ${ }^{8}$ However, a biventricular approach in this patient group may indeed be complicated, with multiple interventions and operations during follow-up as a result of repeated obstructive lesions or even high mortality rates in unsuitable candidates. $^{7,9}$ Therefore, predictive scores have been developed to ease decision making in this subset. ${ }^{1,2}$ These scores, however, have substantial limitations and are less reliable if, for instance, patients lie at a level that is close to the discriminant cut-off value. ${ }^{2}$ Furthermore, these scores are not fully capable of predicting long-term outcome of patients even when the success of uni- or biventricular correction has been predicted accurately. Overall, long-term outcome data, particularly about patients who are managed with biventricular repair, are limited. Additionally, the potential for the eventual development of an adequate size of left ventricular structures that are clearly hypoplastic in the neonatal period is difficult to predict when there is considerable pressure, in the neonatal period, to commit to a definitive surgical strategy.

Alternatively, therapies that aim to avoid major neonatal surgery requiring cardiopulmonary bypass have been proposed, such as hybrid treatment not only for hypoplastic left heart syndrome (HLHS) but also its variants. ${ }^{10}$ This strategy involves bilateral pulmonary artery banding and interventional stenting of the ductus arteriosus (hereafter hybrid stage I) in the neonatal period. Therefore, the eventual performance of a Norwood-type procedure for the aortic arch repair including the superior cavopulmonary connection (Giessen comprehensive stage II operation) or a biventricular correction is deferred until the age of 4 months or 6 to 8 months, respectively. Excellent survival rates after the hybrid stage I have already been reported. ${ }^{6}$ Meanwhile, long-term results of the comprehensive stage II seem promising. In our sample of 154 patients, hybrid stage I and interstage mortality rates are $1.2 \%$ and $6.7 \%$, respectively. Survival in the entire cohort is $77 \%$ at the 15 -year follow-up (unpublished data). One of the main advantages of the hybrid approach is to retain the potential of borderline hypoplastic left hearts to receive a biventricular correction (BVC) until the age of 6 to 8 months. More important, the critical decision on a neonatal procedure can be delayed without compromising the survival of patients by keeping open the option of a uni- or biventricular circulation.

Herein, we report our experience and the long-term outcome in 40 patients with HLHS, HLHC, and variants who received biventricular correction following postnatal palliation with a Giessen hybrid stage I approach.

\section{PATIENTS AND METHODS}

Between June 1998 and June 2013, a total of 154 patients with diagnoses of HLHS, HLHC, and variants received an initial hybrid approach with surgical bilateral pulmonary artery banding and interventional stenting of the arterial duct in the Pediatric Heart Center Giessen in Germany. The ethics committee of the Justus-Liebig University, Giessen, approved this retrospective study.

The surgical and interventional methods for the stage I hybrid approach has been described previously. ${ }^{10,11}$ In short, per our actual modified strategy, newborn infants with an initial diagnosis of HLHS or borderline left ventricular structures receive postnatal hemodynamic stabilization by low-dose prostaglandin infusion or by the hybrid stage I as a highurgency approach. Gross neurologic and further multiorgan assessment is accomplished in our intensive care unit to exclude severe syndromes with prognostic relevance. Surgical off-pump bilateral pulmonary artery banding, normally using a $3.5-\mathrm{mm}$ polytetrafluoroethylene prosthesis (in a normal-weight newborn infant, $3.0 \mathrm{~mm}$ when birth weight $<2.5 \mathrm{~kg}$ ), is performed within 3 to 5 days. Thereafter, interventional percutaneous stenting of the ductus arteriosus using a $7-$ to $10-\mathrm{mm}$ stent is electively instituted, mostly within the next 24 to 48 hours.

Considering the retrospective analysis from the group of 154 patients, a cohort of 33 patients who were determined to have borderline/hypoplastic left ventricular structures and 7 patients with HLHS were amenable to receive a biventricular circulation after a hybrid stage I procedure. These patients were operated between May 1999 and April 2013. Most of this cohort $(n=24)$ met the criteria for a diagnosis of HLHC.

The definition of HLHC in this study includes the following criteria:

- Borderline/obstructive left-sided structures that may involve

- left-ventricular end-diastolic volume of $\leq 20 \mathrm{~mL} / \mathrm{m}^{2}$

- aortic valve size $<5 \mathrm{~mm}$ in a normal-weight full-term newborn infant

- mitral valve to tricuspid valve size ratio of $<0.7$.

- Duct-dependent circulation of the lower body

- Antegrade flow to the ascending aorta up to the coarctation.

The only exception to the definition above is the diagnosis of aortic atresia with the presence of a ventricular septal defect and 2 adequately sized ventricles.

Patient data have been gathered using a retrospective analysis of patient charts, operative reports, and electronically saved echocardiographic images. All patients received echocardiographic evaluation before hybrid stage I and corrective surgery by experienced pediatric cardiologists. Because all patients were monitored with regular visits in our outpatient cardiology clinic, information about long-term morbidity and mortality are available for the entire group. Interventions after the biventricular correction were defined as any surgical or interventional procedure that was required because of a cardiac or hemodynamic problem.

Median age of patients before hybrid stage I was 8.5 days (2-40). Median weight and body surface area before hybrid stage I procedure were $3.0 \mathrm{~kg}$ (1.6-3.8) and $0.2 \mathrm{~m}^{2}(0.13-0.23)$, respectively. These patients $(\mathrm{n}=40)$ had the diagnoses HLHC $(\mathrm{n}=15)$, HLHC with interrupted aortic arch $(\mathrm{n}=9)$, critical aortic stenosis with hypoplastic aortic arch $(n=4)$, imbalanced atrioventricular septal defect with a hypoplastic aortic arch $(\mathrm{n}=2)$, double-outlet right ventricle with a hypoplastic aortic arch $(\mathrm{n}=1)$, double-outlet right ventricle with an interrupted aortic arch $(\mathrm{n}=1)$, and d-transposition of the great arteries with an interrupted aortic $\operatorname{arch}(n=1)$. Furthermore, 7 patients who were born with HLHS with mitral and aortic stenosis (including 1 patient with aortic atresia and a ventricular septal defect) postnatally predestined for univentricular palliation were later on switched to biventricular repair. Discrete or long-segment coarctation of the aorta was present in $21(52.5 \%)$ of the patients. There were no other patients who were initially considered for a biventricular repair and failed to receive a biventricular circulation or were omitted from this analysis. 
TABLE 1. Characteristics of patients who received biventricular correction after hybrid stage I with outcome data

\begin{tabular}{|c|c|c|c|c|c|}
\hline Patient no. & Cardiac diagnosis & Age at BVC (mo) & Type of BVC & Interventions post-BVC & Outcome \\
\hline 1 & HLHC, IAA, VSD & 4.8 & Direct & & A \\
\hline 2 & HLHC, IAA, VSD, hypAoA & 1.6 & Direct & AoA reco. (4 y 6 mo) & A \\
\hline 3 & HLHS (AS, MS) & 9.7 & Direct & & A \\
\hline 4 & iAVSD, AA, hypAoA & 8.7 & Norwood/Rastelli & & A \\
\hline 5 & $\begin{array}{l}\text { HLHS (AS, MS, MI), LV } \\
\text { dysfunction, EFE }\end{array}$ & 6.2 & Ross-Konno & & A \\
\hline 6 & HLHS (AS, MS), coronary fistula & 6.7 & Direct & PMI (4 d) & A \\
\hline 7 & HLHS (AS, MS) & 5.3 & Direct & & A \\
\hline 8 & HLHS (AS, MS) & 5.1 & Direct & HTx (4 mo) & A \\
\hline 9 & HLHC, IAA, VSD & 9.4 & Norwood/Rastelli & RPA stent. (4 y) & A \\
\hline 10 & D-TGA, VSD, hypAV, hyp AoA & 7.2 & Rastelli & & A \\
\hline 11 & HLHC, hypAoA, VSD & 10.8 & Direct & & $\mathrm{D}$ \\
\hline 12 & AS, hypAa & 7.7 & Direct & & A \\
\hline 13 & AS, hypAoA, VSD & 8.1 & Direct & & $\mathrm{D}$ \\
\hline 14 & HLHC, EFE & 9.5 & Direct & & A \\
\hline 15 & HLHC, IAA, VSD & 13.8 & Norwood/Rastelli & & A \\
\hline 16 & HLHC, IAA, VSD & 7.2 & Direct & & A \\
\hline 17 & AS, hypAoA, VSD & 8.3 & Direct & Dilation AoIst (4 y 6 mo) & A \\
\hline 18 & HLHC, IAA, VSD & 5.2 & Direct & & A \\
\hline 19 & HLHS, VSD & 6.6 & Direct & $\begin{array}{l}\text { SAS res. }(1 \text { y } 6 \text { mo }) \\
\text { Ross-Konno }(4 \text { y) }\end{array}$ & A \\
\hline 20 & HLHC, IAA, VSD, hypAoA & 11.0 & Direct & & A \\
\hline 21 & DORV, hypAoA & 4.8 & Arterial switch & & $\mathrm{D}$ \\
\hline 22 & HLHC, VSD, hypAoA & 2.1 & Direct & & A \\
\hline 23 & AS, hypAoA, VSD, RVOTO & 3.5 & Direct & AoA reco. $(8 \mathrm{~d})$ & A \\
\hline 24 & HLHC, VSD, hypAoA & 4.7 & Direct & & A \\
\hline 25 & HLHC, VSD, hypAoA & 6.6 & Direct & RPA reco. (4 d) & A \\
\hline 26 & HLHC, VSD, hypAoA & 8.0 & Direct & & A \\
\hline 27 & $\begin{array}{l}\text { HLHC, IAA, multiple VSD, } \\
\text { hypAoA }\end{array}$ & 6.7 & Direct & & A \\
\hline 28 & HLHC, VSD, hypAoA & 7.6 & Direct & & A \\
\hline 29 & HLHC, hypAoA, RV hypertrophy & 6.5 & Direct & & A \\
\hline 30 & HLHC, hypAoA, EFE & 2.5 & Direct* & TV reco. $(1 \mathrm{mo})$ & $\mathrm{D}$ \\
\hline 31 & iAVSD, hypLV, IAA & 1.7 & $\begin{array}{c}\text { Direct with AV-Canal } \\
\text { correction }\end{array}$ & & A \\
\hline 32 & DORV, IAA, hypAoA & 5.0 & Arterial switch & $\begin{array}{l}\text { LPA/RPA stent. } \\
\text { AoIst stent. ( } 4 \mathrm{mo})\end{array}$ & A \\
\hline 33 & HLHC, VSD, hypAoA & 7.5 & Direct & & A \\
\hline 34 & HLHC, IAA, VSD & 9.0 & Norwood/Rastelli & & A \\
\hline 35 & HLHC, VSD, hypAoA & 13.7 & Direct & SAS res. $(9 \mathrm{mo})$ & A \\
\hline 36 & HLHC, VSD, hypAoA & 2.7 & Direct & & A \\
\hline 37 & HLHC, hypAoA & 11.0 & Direct & & A \\
\hline 38 & HLHS (AS, MS), VSD & 7.4 & Direct & & A \\
\hline 39 & HLHC, VSD, hypAoA & 3.8 & Direct & & A \\
\hline 40 & HLHC, hypAoA, EFE & 7.5 & Direct & Switch to UVC (3 mo) & $\mathrm{A}$ \\
\hline
\end{tabular}

$B V C$, Biventricular correction; $H L H C$, hypoplastic left heart complex; IAA, interrupted aortic arch; $V S D$, ventricular septal defect; $A$, alive; hypAoA, hypoplastic aortic arch; $A o A$, aortic arch; $H L H S$, hypoplastic left heart syndrome; $A S$, aortic stenosis; $M S$, mitral stenosis; $i A V S D$, imbalanced atrioventricular septal defect; $A A$, aortic atresia; $M I$, mitral insufficiency; $L V$, left ventricle; $E F E$, endocardial fibroelastosis; $P M I$, pacemaker implantation; $H T x$, orthotopic heart transplantation; RPA, right pulmonary artery; stent., stenting; $D$-TGA, d-transposition of the great arteries; hypAV, hypoplastic aortic valve; $D$, dead; hypAa, hypoplastic ascending aorta; AoIst, aortic isthmus; SAS, subaortic stenosis; res., resection; $D O R V$, double-outlet right ventricle; $R V O T O$, right ventricular outflow tract obstruction; reco., reconstruction; $R V$, right ventricle; $T V$, tricuspid valve; hyp $L V$, hypoplastic left ventricle; $L P A$, left pulmonary artery; $U V C$, univentricular circulation. *Intraoperative conversion to univentricular palliation.

We intended to keep a restrictive interatrial communication considering further postnatal left ventricular growth potential in patients with a higher probability of biventricular repair. The immediate intensive care course of the patient after stage I would be alarming in case of an overrestrictive atrial septum with the development of out-of-proportion postcapillary pulmonary hypertension and arterial desaturation. A left atrial pressure up to $15 \mathrm{~mm}$ $\mathrm{Hg}$ and a gradient over the interatrial communication between 5 and 10 $\mathrm{mm} \mathrm{Hg}$ seemed to be tolerable in this setting.

Table 1 shows characteristics of patients who have received a biventricular correction. Figure 1 presents the spectrum of diagnoses in this patient group. 


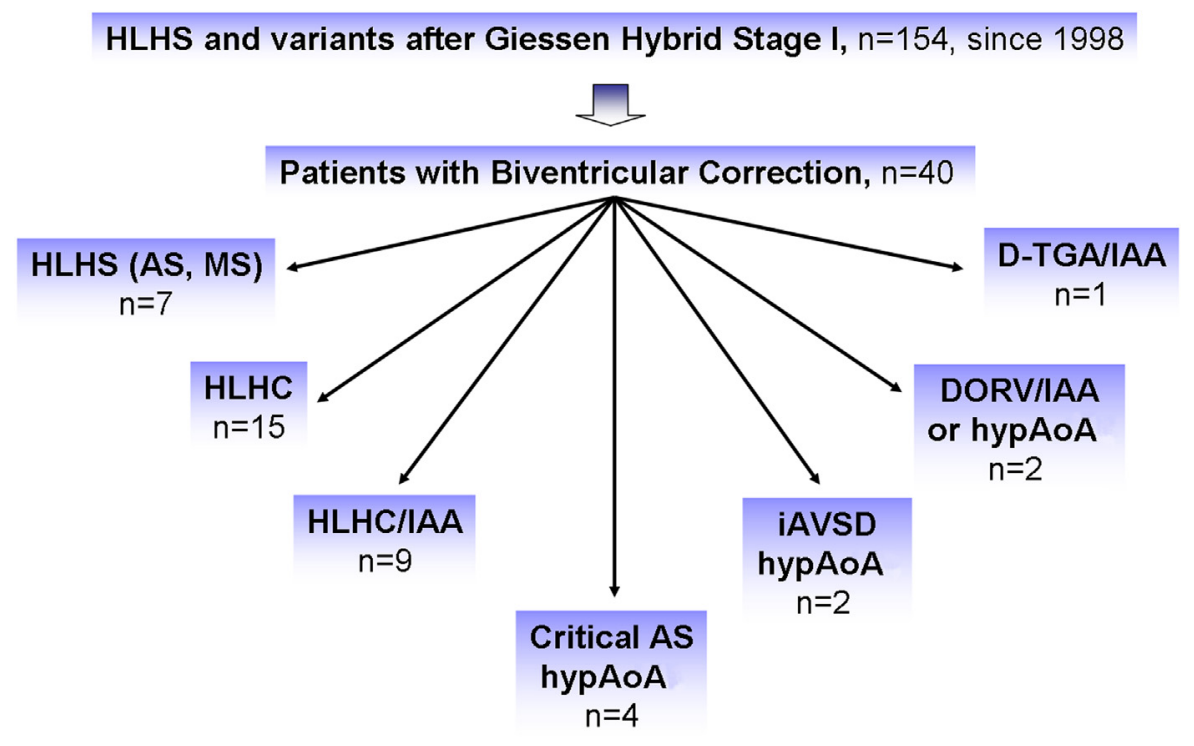

4 patients $(10 \%)$ with EFE

21 patients (52.5\%) with discrete or long segment CoA

FIGURE 1. Hypoplastic left heart diagnoses within the group of the patients who received biventricular correction. HLHS, Hypoplastic left heart syndrome; $A S$, aortic stenosis; $M S$, mitral stenosis; $D-T G A$, d-transposition of the great arteries; IAA, interrupted aortic arch; HLHC, hypoplastic left heart complex; DORV, double-outlet right ventricle; hypAoA, hypoplastic aortic arch; $i A V S D$, imbalanced atrioventricular septal defect; $E F E$, endocardial fibroelastosis; $C O A$, coarctation.

\section{Echocardiographic Measurements}

Electronically saved echocardiographic studies of all patients were reviewed without the knowledge of each patient's outcome. Two echocardiographic studies that had been performed before hybrid stage I and before biventricular correction were included in the analysis. The post-hybrid stage I echocardiography was derived from available echocardiography reports, images, and sequences. In order to avoid bias, a single echocardiographer performed an actual repeat analysis and recorded the measurements from the electronically available images and sequences. Besides categorical variables, measurements of morphometric parameters were performed offline. Annular diameter of the aortic valve was measured in systole from the parasternal long-axis view. Left ventricular and right ventricular enddiastolic lengths were measured from the apical 4-chamber view. These were defined as the direct distance from the middle of each atrioventricular valve to the most apical endocardial extension of each ventricle. Left ventricular to right ventricular length ratio was calculated for each patient. End-diastolic mitral valve diameter was measured from the 4-chamber view. Discriminant scores were calculated retrospectively for each patient based on the echocardiograms before hybrid stage I and biventricular correction according to Colan and colleagues. ${ }^{2}$

\section{Surgical Techniques for Biventricular Correction}

Median age of patients at the time of biventricular correction was 6.7 months (1.6-13.8). Median weight and body surface area was $6.0 \mathrm{~kg}$ (3.0-9.6) and $0.32 \mathrm{~m}^{2}(0.18-0.43)$, respectively. Standard surgical procedures during biventricular correction included bilateral pulmonary artery debanding, stent removal from the ductus arteriosus with reconstruction of the pulmonary artery on the site of the stent and reconstruction of the aortic arch. Pulmonary arteries were reconstructed using autologous pericardium if needed at the sites of the bilateral bandings. During the last few years, a simple Hegar dilation or intraoperative balloon dilation was mostly adequate for the relief of narrowing on the pulmonary arteries due to bandings. Selective cerebral perfusion by direct or prosthesis-aided cannulation of the brachiocephalic trunk with moderate hypothermia at $28^{\circ} \mathrm{C}$ to $30^{\circ} \mathrm{C}$ was instituted in all patients. In patients with adequate size of the ascending aorta during biventricular repair, selective myocardial perfusion was added to the circuit except during intracardiac repair (in this cohort, 6 patients, 15\%). Aortic arch reconstruction was performed using a preformed curved xenopericardial patch/conduit (BioIntegral Surgical, Inc, Canada) after the year 2002 because of our experience with the use of aortic and pulmonary homografts for aortic reconstruction showing extensive calcifications at Fontan completion. This is a commercially available curved patch specifically designed for a use in Norwood-type procedures. With the No-React (BioIntegral Surgical) treatment (heparin-based proprietary detoxification and biomodification of glutaraldehyde-treated tissue that stabilizes tissue cross-linking and prevents release of aldehydes), this patch is free of calcification at Comprehensive Stage II and Fontan completion.

The applied surgical procedures during the biventricular correction and the number of treated patients are as follows: direct biventricular correction including repair of intracardiac defects $(\mathrm{n}=32)$, Norwood/Rastelli (Yasui) operation $(n=4)$, arterial switch operation $(n=2)$, Rastelli operation $(\mathrm{n}=1)$, and Ross-Konno procedure with ascending aortic/aortic arch reconstruction $(\mathrm{n}=1)$. Concomitantly, we performed ventricular septal defect (VSD) closure in 27 cases $(67.5 \%)$, VSD enlargement in 2 cases $(5 \%)$, reconstruction of the branch pulmonary arteries in 20 cases $(50 \%)$, right ventricular outflow tract reconstruction in 6 cases $(20 \%)$, subaortic resection in 3 cases $(7.5 \%)$, aortic valve reconstruction in 3 cases $(7.5 \%)$, mitral valve reconstruction in 5 cases $(12.5 \%)$, tricuspid valve reconstruction in 2 cases $(5 \%)$, and closure of a coronary fistula in 1 case $(2.5 \%)$. An atrial septal communication of 2.8 to $4 \mathrm{~mm}(\mathrm{n}=10)$ and a ventricular septal communication $(n=1)$ were left open in 11 cases $(27.5 \%)$.

\section{Statistical Analysis}

All data were stored and analyzed with the SPSS software package (version 15.0; SPSS Inc, Chicago, Ill). Descriptive statistics were computed for variables of interest. 
Data are presented as median (range) in most cases and otherwise specified. In dependence of distributions, the paired t test or the Mann-Whitney $U$ test was used for comparison within the groups between 2 time points. $\mathrm{Z}$ scores were calculated for each patient using a noncommercial software that was based on former publications with nomograms. ${ }^{12,13}$ Actuarial survival and freedom from intervention after biventricular correction were calculated using the Kaplan-Meier equation. A $P$ value of $<.05$ was considered significant.

\section{RESULTS}

All patients survived the hybrid stage I procedure. Figure 2, A, shows Kaplan-Meier survival analysis for the entire group.

\section{Mortality}

Table E1 and Appendix E1 show characteristics of patients who did not survive biventricular correction. Median survival time after biventricular correction is $7.9(0.9-14.9)$ years. Overall mortality was $10 \%$ (4 patients) during the follow-up. These occurred 4 weeks, 5 weeks, 6 weeks, and 4 months after biventricular correction, respectively. Early mortality occurred in only 2 cases before hospital discharge.

\section{Interventions After Biventricular Correction}

The type and timing of the interventions after biventricular correction are listed in Table 1. Figure 2, B, shows freedom from intervention during the follow-up after biventricular correction.

A total of 16 interventions in $12(30 \%)$ patients had to be performed during the entire follow-up. From these cases, 10 were surgical procedures: 1 late switch to univentricular circulation in a patient with HLHC and endocardial fibroelastosis, 1 orthotopic heart transplantation, 2 aortic arch re-reconstructions; surgical interventions on the left ventricular outflow tract had to be performed in 3 patients: 1 Ross-Konno procedure and 2 subaortic membrane resections. Further interventions included 1 pulmonary vein dilation, 1 aortic arch dilation, 1 right pulmonary artery reconstruction, 1 tricuspid valve reconstruction, and 1 early pacemaker implantation due to high-grade atrioventricular block. In 4 cases, interventional procedures with either simple balloon dilation or stent placement in the aortic isthmus or pulmonary vessels were required.

\section{Development of Left Ventricular Structures}

Figure 3 presents the development of left ventricular structures (absolute values and $\mathrm{Z}$ scores) in comparison with the measurements at the time of hybrid stage I and biventricular correction.

The absolute diameter of the aortic valve increased from a median of $0.55 \mathrm{~cm}(0.30-0.80)$ at hybrid stage I to $0.7 \mathrm{~cm}$ (0.3-0.9) before biventricular correction. The mitral valve annulus was $1.2 \mathrm{~cm}(0.65-1.70)$ at hybrid stage I and increased to $1.5 \mathrm{~cm} \quad(0.9-2.0)$ before biventricular correction.

The median aortic valve $\mathrm{Z}$ score was -2.13 ( -7.14 to $0.77)$ before the performance of hybrid stage I and developed to a median $\mathrm{Z}$ score of $-1.21(-8.87$ to -0.08$)$ before the performance of biventricular correction $(P=.04)$. A less pronounced trend was observed in mitral valve $\mathrm{Z}$ score, for which the median was 0.52 ( -2.97 to 2.42$)$ at stage I and was found to be 0.98 ( -2.35 to 2.64 ) before biventricular correction $(P=.05)$.

The median ratio of left ventricular to right ventricular length was $0.90(0.69-1.10)$ at hybrid stage I and found to be $0.92(0.73-1.33)$ before biventricular correction (Figure 4, A).

The application of the discriminant model that was published by Colan and colleagues ${ }^{2}$ in 2006 revealed a median score of -0.72 ( -2.80 to 1.75 ) for our group before the performance of hybrid stage I procedure. A repeated calculation before the biventricular correction showed that the same cohort had a median score of $1.47(-2.43$ to 3.79 ; $P<.001$ ) (Figure 4, $B$ ). The cutoff for an accurate prediction of outcome was postulated to be -0.65 by the authors. Before hybrid stage I, 18 patients in our cohort were above this level, and so a biventricular correction seemed to be feasible for this group; however, before biventricular correction, the discriminant scores of 35 patients were calculated to be above the critical level of -0.65 .
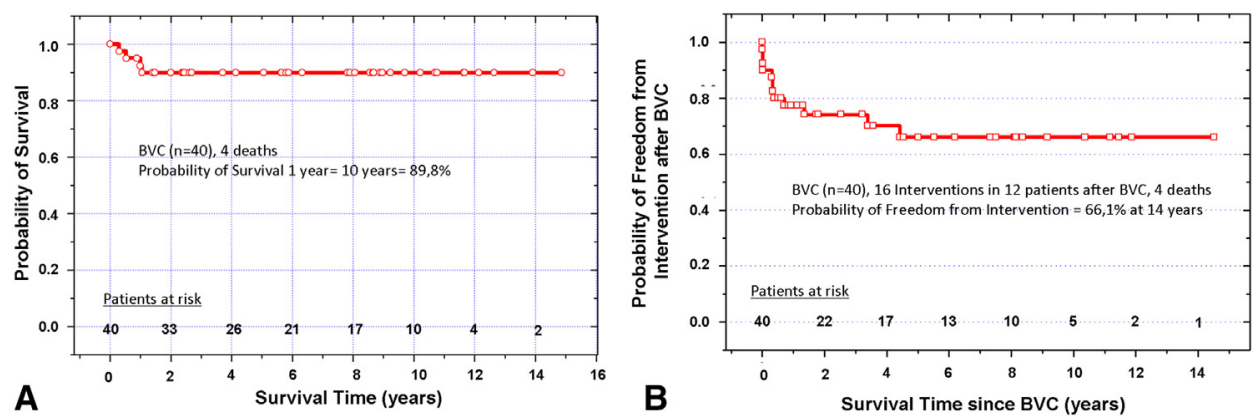

FIGURE 2. A, Kaplan-Meier survival analysis for patients with HLHC and variants after biventricular correction. B, Freedom from subsequent surgery or intervention for patients after biventricular correction. $B V C$, Biventricular correction. 

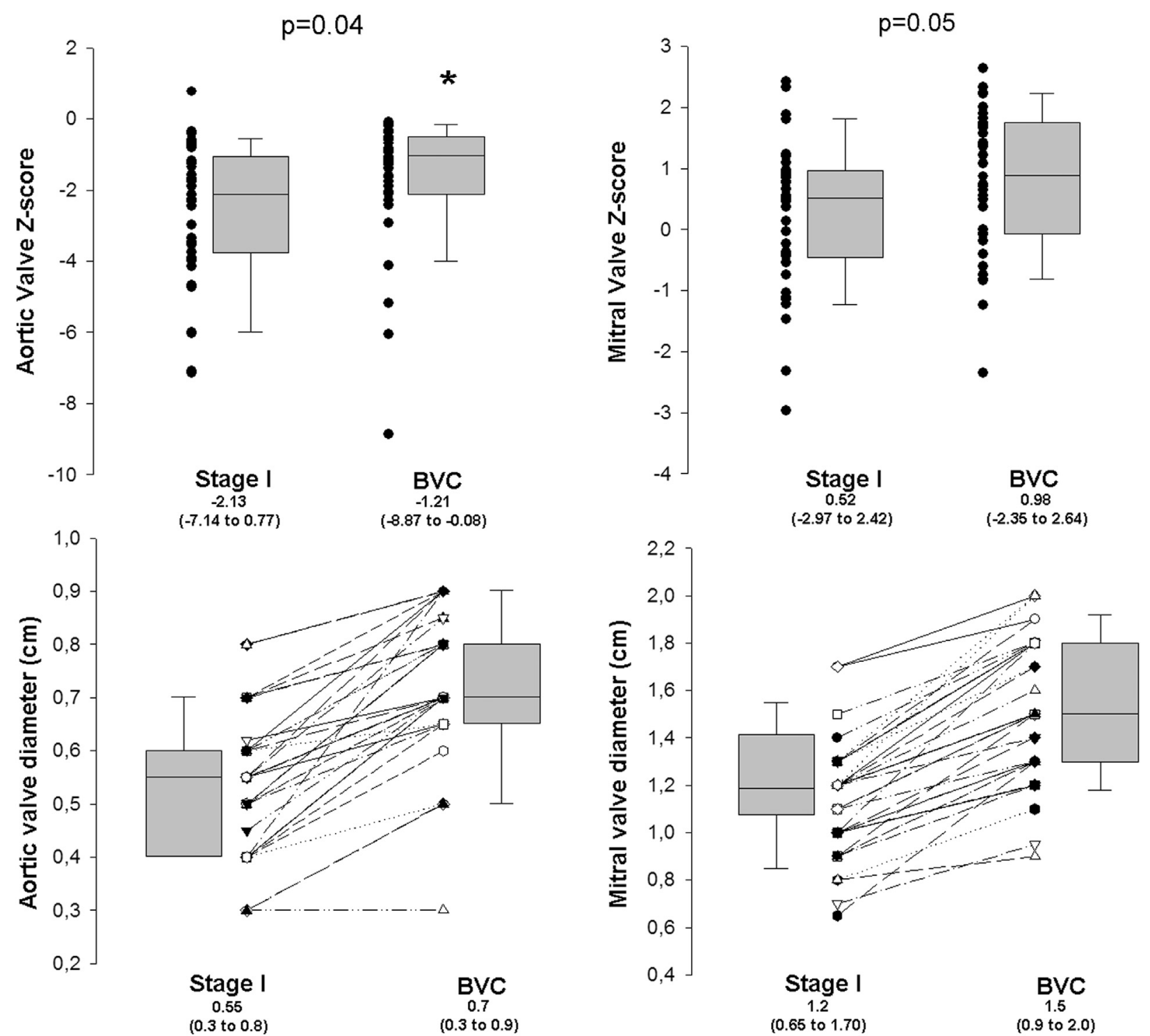

FIGURE 3. Scatter and box plots showing the development of aortic and mitral valve $Z$ scores and their diameters in comparison of time points before hybrid stage I and before biventricular correction. Numbers below the plots represent the median (minimum and maximum) for each parameter. Each patient is symbolized with filled circle in the upper 2 figures. In the lower figures, different types of symbols (filled/unfilled and shapes) are used to identify patients before stage I, and the values correspond to the measurements before biventricular correction. Stage I, Hybrid stage I; $B V C$, biventricular correction. *P<.05.

\section{DISCUSSION}

Management of the "small left heart" depends on the degree of underdevelopment of the left ventricle and/or its components. The wide spectrum of variable degrees of underdevelopment of left heart structures may complicate the critical strategic decision of pursuing a uni- or biventricular approach. The imminent question remains whether the left ventricle is capable of sustaining the systemic circulation when such a baby is presented in the neonatal period. A correct and timely strategic decision is needed, particularly for the intermediate group so as to achieve an optimal outcome when a primary major surgery has to be performed in the neonatal period. Alternatively, an initial hybrid approach with bilateral pulmonary artery banding and ductal stenting may be performed without the need of an early decision and major surgery. ${ }^{10}$

Besides predictive scores for the estimation of success with either a uni- or biventricular approach, simple echocardiographic measurements - such as critical levels of an indexed mitral valve area of $4.75 \mathrm{~cm}^{2} / \mathrm{m}^{2}$, left ventricular length to heart long axis ratio of 0.8 , or an indexed aortic root size of $3.5 \mathrm{~cm} / \mathrm{m}^{2}$-have been postulated as predictors of outcome after biventricular repair. ${ }^{1}$ However, none of these parameters serves as a guidepost alone for a correct decision making in the neonatal period, and especially a wrong decision to establish a biventricular circulation in a patient with a borderline but insufficient left ventricular size and/or function may 

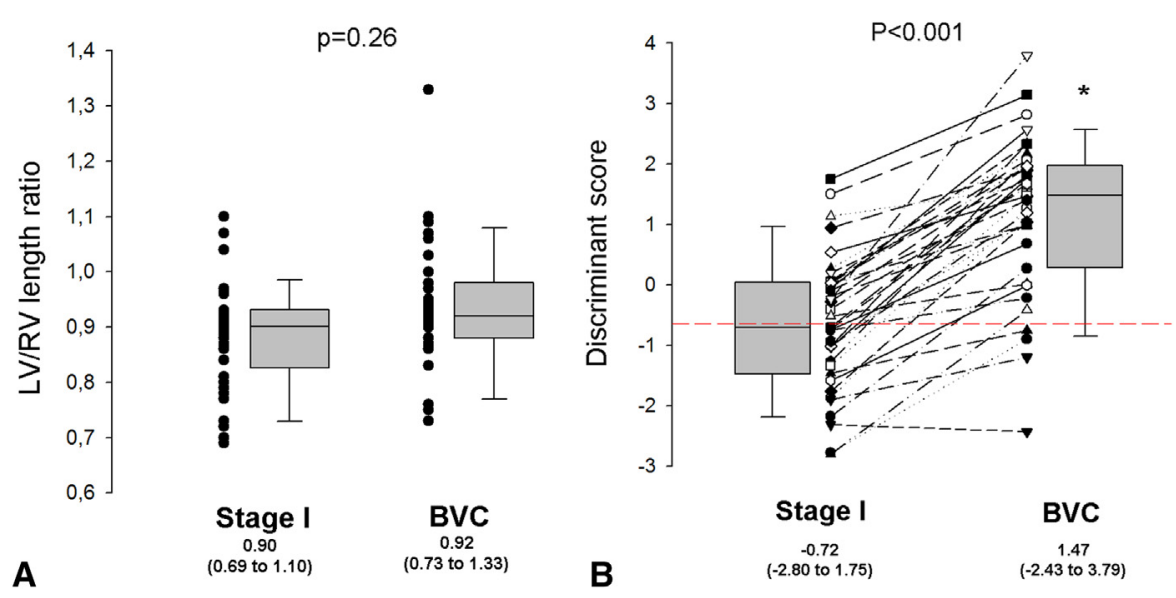

FIGURE 4. LV/RV length ratio (A) and application of the discriminant score (B) by Colan and colleagues ${ }^{2}$ to our cohort before hybrid stage I and before biventricular correction. Red line represents the critical cutoff of -0.65 above which a biventricular correction is favorable. Numbers below the plots indicate the median (minimum and maximum) for each parameter. Each patient is represented by a filled circle in (A). In (B), different types of symbols (filled/ unfilled and shapes) are used to identify patients before stage I and the values correspond to the measurements before biventricular correction. $L V$, Left ventricle; $R V$, right ventricle; Stage I, hybrid stage I; $B V C$, biventricular correction. $* P<.05$.

lead to an extremely unfavorable outcome. Furthermore, it is also important to mention that predictive calculations reach their limits of accuracy in patients who belong to the intermediate group of left heart hypoplasia, and these scores fail to estimate the long-term effects of any strategy.

Because all of our patients with HLHS, HLHC, and variants received an initial hybrid approach, patients in this nonselected cohort belong to a wide spectrum of hypoplastic left heart variants similar to the usual clinical setting. However, most of the patients had the diagnosis of a hypoplastic left heart complex, which is defined as a ductdependent lower body circulation but almost always an antegrade flow through the ascending aorta with borderline left heart structures.

Usually we first perform bilateral pulmonary artery banding, which is followed by ductal stenting in the catheterization laboratory within the next 24 to 48 hours via a transfemoral approach. The main reason for this alternative approach is to delineate the precise anatomy of the aortoductal junction, which varies considerably case by case in the catheterization laboratory instead of in the operation theater. Additionally, a wide variety of stents is available in Europe, in particular, self-expandable stents CE marked for ductal stenting in newborn infants, which can be placed according to the unique anatomy of the aorto-ductal region. Another advantage of this approach is to actively prevent inadvertent placement of the ductal stent too far into the descending aorta. This problem has already been described from centers that combine bilateral banding and ductal stenting in the operating room. The third, but more important, fact is that (about 30\%) in newborn infants with HLHS, there may be a requirement for a manipulation of the atrial septal communication, which may be performed concomitantly but prior to ductal stenting.
By pursuing the Giessen hybrid approach and subsequent operation for a uni- or biventricular approach at the age of 4 to 8 months, there was no mortality after hybrid stage I in our cohort. Overall, 4 patients died after biventricular correction. One patient with HLHS (severe aortic and mitral hypoplasia) and endocardial fibroelastosis after having received the initial hybrid stage I in our center had to be switched to a univentricular circulation after the performance biventricular correction resection of endocardial fibroelastosis in another center. He is now alive after Fontan completion.

Surgical reinterventions ( 10 operations) had to be performed in 8 patients $(20 \%)$ after biventricular correction. Higher rates of failure and reoperations have been reported in similar cohorts with early biventricular corrective surgery. The most frequent reason for a reoperation seems to be obstructions in the left ventricular outflow tract even with adequate growth of the left heart observed during follow-up. ${ }^{14}$ In our group, this occurred in 3 cases, including 1 Ross-Konno operation and 2 resections of a subaortic membrane.

Recent reports have also claimed high frequency of the need for pulmonary artery interventions following hybrid therapy with bilateral pulmonary artery banding. ${ }^{15} \mathrm{We}$ cannot confirm this finding because only 3 patients needed an intervention with stenting or a reoperation due to pulmonary artery problems following biventricular repair. The reason may lie in the fact that pulmonary artery narrowing if existent was addressed during biventricular repair either with patch enlargement or intraoperative balloon dilation.

Growth of left ventricular structures after early biventricular correction or neonatal aortic valve intervention has been shown by others. ${ }^{14,16}$ In our study, we showed an increase of aortic valve and mitral diameters and $\mathrm{Z}$ scores at the time point of biventricular correction in comparison to the measurements before hybrid stage I. A comparable tendency 
was present in the measurement of left ventricular to right ventricular inflow length ratio. However, the left ventricular inflow length $\mathrm{Z}$ score was slightly below the value of the measurement before hybrid stage I. This is an interesting finding because it may mean that even with a borderline left ventricular cavity in the echocardiographic measurement, a biventricular correction can be established with good outcome in this group. The prerequisite for this success seems an adequate growth of the aortic valve and corrective surgery by addressing all obstructive lesions in the left ventricle-aortic bloodstream. This fact has already been described in the refined Rhodes score, which improved the accuracy of the discriminant model when aortic annulus $\mathrm{Z}$ score was included in the equation instead of the aortic root diameter. ${ }^{2}$ Further groups suggested unfavorable results for patients with ventriculo-aortic junction of less than $5 \mathrm{~mm}$, a left ventricular inflow dimension of $25 \mathrm{~mm}$, and a mitral valve diameter of $9 \mathrm{~mm} .{ }^{17}$ In our series, 20 patients had an aortic valve diameter of $5 \mathrm{~mm}$ or less (1 death with syndromic features and aortic valve diameter of $4 \mathrm{~mm}$ ), 17 patients had a left ventricular length of $25 \mathrm{~mm}$ or less, and 7 patients had a mitral valve diameter of $9 \mathrm{~mm}$ or less. Left ventricular to right ventricular inflow length ratio below 0.8 has also been postulated as a critical level favoring a univentricular approach. ${ }^{1} \mathrm{We}$ had 9 patients with an left ventricular to right ventricular length ratio of 0.8 or less in our series. From this subgroup, 1 patient who had endocardial fibroelastosis died after early conversion to univentricular circulation because of several episodes of bacterial pneumonia, liver failure with severe coagulation derangement, and shunt thrombosis 5 weeks postoperatively. If the application of the discriminant score to our group would have been accepted as a guide for the decision in the neonatal period to pursue a univentricular or biventricular strategy, only 18 patients would lie above the postulated cutoff of -0.65 favoring biventricular correction. This number of patients has almost doubled before we performed biventricular correction, with 35 patients having a score higher than -0.65 . The change in the discriminant score was statistically significant. This finding again proves the advantage of the hybrid approach, which allows for a potential growth of the left-sided structures when a discriminant score is to be instituted for the decision. As stated by the authors of the original paper, the application of the score in a group of borderline patients may not reveal as accurate discrimination of outcome as when it is applied to patients at the extremes. This finding is important, strongly pronouncing the challenges in an intermediate group of patients.

Schwartz and colleagues ${ }^{3}$ retrospectively analyzed 72 patients with more than 2 left ventricular obstructive lesions or hypoplasia who underwent biventricular correction without former catheter or surgical intervention. They postulated among other factors the presence of a moderate or large ventricular septal defect as a strong predictor of failure after biventricular correction even with a correlation of increasing size of the ventricular septal defect and aortic valve without a reasonable explanation. In our series, 27 patients had a ventricular septal defect, and 3 of them belong to patients who died after biventricular correction. The same study showed that an antegrade flow through the ascending aorta was a risk factor for biventricular correction, which is in contrast to our perspective and experience because the majority of our patients had antegrade flow through the ascending aorta documented by Doppler echocardiography.

The presence of endocardial fibroelastosis was declared as a risk factor for the outcome by impairing left ventricular diastolic function. ${ }^{18,19}$ In our series, 4 patients had endocardial fibroelastosis. One of these had to be converted to univentricular circulation after biventricular correction; another patient with accompanying psychomotor development retardation received a Ross-Konno procedure during biventricular correction and developed postcapillary pulmonary hypertension but is doing well with conservative medical therapy at the 11-year follow-up. As mentioned above, 1 other patient with systemic syndrome and endocardial fibroelastosis died 5 weeks postoperatively. A former 2.5$\mathrm{kg}$ newborn infant with HLHC and endocardial fibroelastosis is doing well 9 years after biventricular correction. We also do believe that the presence and grade of endocardial fibroelastosis has to be taken cautiously into account by pursuing the biventricular pathway.

In conclusion, the hybrid strategy emerges for patients with HLHS and variants especially with borderline left ventricle fulfilling the criteria of HLHC as a reasonable alternative to conventional strategy with direct biventricular treatment or neonatal performance of a Norwood-type palliation. This is the first study to analyze long-term outcome in patients with borderline left ventricle who have been treated with initial hybrid strategy and subsequently corrected with biventricular circulation. The advantages of this approach include delaying the critical decision/operation for a univentricular palliation or a biventricular correction without compromising but potentially improving survival of these patients. Hence, allowing time for the growth potential of the left ventricle avoiding a major surgery in the neonatal period, we can keep both uni- and biventricular pathways as alternatives at the age of 4 to 8 months with satisfactory long-term outcome. Our experience suggests following important factors in achieving a favorable outcome for patients with borderline left ventricular structures who should receive biventricular correction: first, hybrid treatment with intense perioperative collaboration with pediatric cardiology; second, relief of all left-sided obstructing lesions and when needed creation of an interatrial septal communication during biventricular repair; and third, consequent follow-up of patients, including liberalized indication for cardiac magnetic resonance imaging (available in our center since 2008) for the assessment of left ventricular structures before comprehensive stage II or biventricular correction. 


\section{Limitations of the Study}

The limitations of the study are its retrospective design, reassessment of echocardiographic data from original but electronically saved echocardiographic sequences, lack of definitive criteria in the beginning of the series to define the diagnostic group of patients (eg, HLHS vs HLHC vs aortic stenosis with hypoplastic aortic arch), which had to be completed in the retrospective reevaluation. The technical quality of the echocardiograms varied but were not absolutely limiting in acquiring the desired measurements. Only limited but essential echocardiographic data have yet been acquired. The developments of further echocardiographic parameters such as indexed mitral valve area, left ventricular end-diastolic volume, or aortic root diameter, etc and their potential effects on the outcome cannot be shown with the available data.

You can watch a Webcast of this AATS meeting presentation by going to: http://webcast.aats.org/2014/files/ Monday/20140428_415pm_435pm_Can_Yerebakan.mp4.

\section{References}

1. Rhodes LA, Colan SD, Perry SB, Jonas RA, Sanders SP. Predictors of survival in neonates with critical aortic stenosis. Circulation. 1991;84:2325-35.

2. Colan SD, McElhinney DB, Crawford EC, Keane JF, Lock JE. Validation and reevaluation of a discriminant model predicting anatomic suitability for biventricular repair in neonates with aortic stenosis. J Am Coll Cardiol. 2006;47:1858-65.

3. Schwartz ML, Gauvreau K, Geva T. Predictors of outcome of biventricular repair in infants with multiple left heart obstructive lesions. Circulation. 2001;104: 682-7.

4. Ohye RG, Sleeper LA, Mahony L, Newburger JW, Pearson GD, Lu M, et al. Comparison of shunt types in the Norwood procedure for single-ventricle lesions. N Engl J Med. 2010;362:1980-92.

5. Iannettoni MD, Bove EL, Mosca RS, Lupinetti FM, Dorostkar PC, Ludomirsky A, et al. Improving results with first-stage palliation for hypoplastic left heart syndrome. J Thorac Cardiovasc Surg. 1994;107:934-40.

6. Galantowicz M, Cheatham JP, Phillips A, Cua CL, Hoffman TM, Hill SL, et al. Hybrid approach for hypoplastic left heart syndrome: intermediate results after the learning curve. Ann Thorac Surg. 2008;85:2063-70.

7. Tchervenkov CI, Tahta SA, Jutras LC, Beland MJ. Biventricular repair in neonates with hypoplastic left heart complex. Ann Thorac Surg. 1998;66:1350-7.

8. de Leval MR. The Fontan circulation: What have we learned? What to expect? Pediatr Cardiol. 1998;19:316-20.

9. Quaegebeur JM, Jonas RA, Weinberg AD, Blackstone EH, Kirklin JW. Outcomes in seriously ill neonates with coarctation of the aorta. A multiinstitutional study. J Thorac Cardiovasc Surg. 1994;108:841-51.

10. Akintuerk H, Michel-Behnke I, Valeske K, Mueller M, Thul J, Bauer J, et al. Stenting of the arterial duct and banding of the pulmonary arteries: basis for combined Norwood stage I and II repair in hypoplastic left heart. Circulation. 2002; 105:1099-103.

11. Akinturk H, Michel-Behnke I, Valeske K, Mueller M, Thul J, Bauer J, et al. Hybrid transcatheter-surgical palliation: basis for univentricular or biventricular repair: the Giessen experience. Pediatr Cardiol. 2007;28:79-87.

12. Daubeney PE, Blackstone EH, Weintraub RG, Slavik Z, Scanlon J, Webber SA. Relationship of the dimension of cardiac structures to body size: an echocardiographic study in normal infants and children. Cardiol Young. 1999;9:402-10.

13. Zilberman MV, Khoury PR, Kimball RT. Two-dimensional echocardiographic valve measurements in healthy children: gender-specific differences. Pediatr Cardiol. 2005;26:356-60

14. Serraf A, Piot JD, Bonnet N, Lacour-Gayet F, Touchot A, Bruniaux J, et al. Biventricular repair approach in ducto-dependent neonates with hypoplastic but morphologically normal left ventricle. J Am Coll Cardiol. 1999;33:827-34.

15. Davies RR, Radtke WA, Klenk D, Pizarro C. Bilateral pulmonary arterial banding results in an increased need for subsequent pulmonary artery interventions. $J$ Thorac Cardiovasc Surg. 2014;147:706-12.
16. Han RK, Gurofsky RC, Lee KJ, Dipchand AI, Williams WG, Smallhorn JF, et al. Outcome and growth potential of left heart structures after neonatal intervention for aortic valve stenosis. J Am Coll Cardiol. 2007;50:2406-14.

17. Leung MP, McKay R, Smith A, Anderson RH, Arnold R. Critical aortic stenosis in early infancy. Anatomic and echocardiographic substrates of successful open valvotomy. J Thorac Cardiovasc Surg. 1991;101:526-35.

18. Gundry SR, Behrendt DM. Prognostic factors in valvotomy for critical aortic stenosis in infancy. J Thorac Cardiovasc Surg. 1986;92:747-54.

19. Mocellin R, Sauer U, Simon B, Comazzi M, Sebening F, Buhlmeyer K. Reduced left ventricular size and endocardial fibroelastosis as correlates of mortality in newborn and young infants with severe aortic valve stenosis. Pediatr Cardiol. $1983 ; 4: 265-72$.

\section{Discussion}

Dr Kristine Guleserian (Dallas, Tex). Thank you very much. I have no disclosures.

I would like to thank you, Dr Yerebakan, and your colleagues from Giessen for a very nice presentation of a novel strategy to achieve biventricular repair for hypoplastic left heart variants, the majority of which in your series were hypoplastic left heart complex, after primary hybrid palliation.

This approach is in contrast to the left ventricular recruitment strategy reported by the Boston group in which the circulation is initially supported with standard single-ventricle palliation while staged procedures are subsequently undertaken to relieve inflow and outflow tract obstruction and resect endocardial fibroelastosis when present in order to promote blood flow through the left ventricle.

Clearly the determination of the feasibility and long-term success of biventricular repair for borderline left ventricle is complex and must include a very thoughtful assessment of morphometric and functional parameters, hemodynamic data, surgical options, and perhaps, most importantly, results in individual and institutional experience.

We always want to make a correct decision and the use of scoring systems or the application of the discriminant model to which you referred is certainly helpful but may not always be accurate in cases that may be at the "cutoff" or for a heterogeneous patient population.

I have three questions for you, but first I would like to clarify one thing for the audience:

Do you perform any primary Norwood procedures at your institution or are you exclusively performing hybrid procedures for all hypoplasts and their variants?

Dr Can Yerebakan (Giessen, Germany). I must confess I am exposed to this new therapy, hybrid therapy, since last year when I joined the group in Giessen. My first experience with congenital heart surgery was during my fellowship in the team of Dr Richard Jonas in Washington, DC, where I could see this classic treatment of Norwood palliation. In Giessen, we do not ever perform the classic Norwood operation. So the presented group and also the hypoplastic left heart syndrome group is a group of patients that is not preselected. So this is real life in Giessen: every patient with a borderline left heart or hypoplastic left heart syndrome is directed to the hybrid procedure either with initial stabilization or with urgent Stage I procedure.

Dr Guleserian. Thank you for that clarification.

Based on your predictions, the majority of the 40 patients identified underwent successful biventricular repair. And I think it is 
fair to say that we oftentimes learn more from our failures than our successes, so I wonder in hindsight whether you uncovered any predictors of these less good outcomes-namely deathsfollowing biventricular repair in those 4 initial nonsurvivors as well as in the 2 patients who underwent transition to univentricular physiology or transplantation.

Dr Yerebakan. I think I cannot give you any statistical analysis on that. But as we saw, the presence of endocardial fibroelastosis seems to be an important predictor, which is also clearly described in the literature. As I said, I have no statistics on that. There were only 4 patients with endocardial fibroelastosis; probably this number is too low to create any significance. Nevertheless, syndromic patients too seem to be at risk. Two of the nonsurvivors in this group had systemic syndromes. And I think in these patients with borderline left ventricle, we follow the strategy of keeping the atrial septum quite restrictive if possible.

Dr Guleserian. Thank you-that nicely leads into my next question.

Dr Yerebakan. And we try to keep the atrial septum quite restrictive in order to promote growth of the left ventricle structures. Patients who do not tolerate this situation most probablythis, of course, requires an analysis - are patients who are turned toward a univentricular pathway.

Dr Guleserian. So that leads into my next question, and that is, you left an atrial septal communication in about $25 \%$ and, in fact, the defect ranged from-

Dr Yerebakan. This is during biventricular correction. I was referring to stage I. At stage I, we try to keep the atrial septum restrictive-we do not open the atrial septum intentionally as far as the patients tolerate it. So, we observe the patient within the next few days in the intensive care unit and then decide whether to proceed with an interventional procedure.

Dr Guleserian. But in this series, when you did your biventricular repair, about $25 \%$ still had an atrial septal communication ranging from 2.8 to $4 \mathrm{~mm}$. And as we know, optimal atrial septal restriction has achieved balance between left ventricular throughput and left atrial hypertension. So in any of those patients, did you have to enlarge the atrial septal defect?

Dr Yerebakan. No, there was no case we had to enlarge.

Dr Guleserian. As for my last question, you have nicely described the common and expected surgical and catheter-based interventions necessary in the group following biventricular repair, so I wonder whether any surgical or catheter-based interventions were necessary following the hybrid procedure but before the biventricular repair? In other words, were any interventions for the branch pulmonary artery bands or ductal stent necessary?

Dr Yerebakan. There were 20 interventions between stage I and stage II that were performed in these 40 patients. Of course, we had to consider the learning curve, because this therapy is performed in Giessen since 1998. In the beginning, second stent placement in the ductus and dilation of the stents in the ductus region were interventions that were performed occasionally. No surgical but percutaneous interventions were performed between stage I and biventricular corrections.

Dr Giovanni Stellin (Padova, Italy). Congratulations for your results. In your experience, you have included 6 patients with interrupted aortic arch and atrial septal defect. In that model, usually the right ventricle is the normal size. So I think it is inappropriate to speak about hypoplastic left heart complex when you have a normal left ventricle. So I wonder whether those 6 patients should be probably excluded in your experience and be another topic of patients.

Dr Yerebakan. Whether these patients should be excluded, am I right?

Dr Stellin. Yes. Because there is a normal left ventricle, so you cannot really talk about a hypoplastic left heart complex. In this instance, the left ventricle has to be hypoplastic. Well, you have a ventricular septal defect; most likely, the left ventricle is normal, so you should probably have excluded those patients.

Dr Yerebakan. Then I am right and may refer to the literature and our experience. I must say the clear line between hypoplastic left heart complex and the more severe or milder spectrum is pretty hard to determine. I think it is a combination of left heart obstructive lesions or small left-sided structures that preclude going straight to a biventricular correction. So you can add these patients if you want.

Dr Stellin. Well, there is a clear definition that has been made in an article, which was written by Christo Tchervenko, and it clearly defined the hypoplastic left heart complex. So in the alternative, you should call it something else.

Dr Christopher A. Caldarone (Toronto, Ontario, Canada). That was a very nice presentation. What you are describing is a surgical cohort selected from the group of patients who underwent bilateral pulmonary artery banding and ductal stenting. I think we would be interested to know though about all the patients in whom you used the strategy to defer a 1-ventricle/2-ventricle decision. Presumably, there were some other patients who you thought might be suitable for 2-ventricle repair but did not. So how successful is this strategy when you a priori identify that the indication for the procedure is a 1-ventricle or 2-ventricle decision deferral. That is question No. 1.

Question No. 2, can you be more specific with regard to your management of the atrial septum. Specifically, what left atrial pressure by echo gradient would you be willing to tolerate, and were there any patients in whom that gradient got too big? You did an atrial septostomy and then took them out of the running for 2ventricle repair, in which case they would not have been reported here.

Dr Yerebakan. With regard to your first question, we do not do this analysis a priori, so we do not decide whether a patient is going to have a univentricular or biventricular repair. This is decided only when we had the opportunity at the age of 4 to 8 months, as I said, and a median of 6.7 months. So the decision is made basically on the growth of the left ventricle structures and on the experience of the group as to which patients would tolerate a biventricular correction. So in order to say that, we had to make a prior analysis before going to biventricular repair, and I do not have those data.

This study is a continuing study, and a retrospective one, so I cannot tell you about exact numbers where one would say left ventricular pressure is that and we would go ahead and open the atrial septum. In these patients-you speak about stage I, right-so after Stage I there was no requirement in this group to enlarge the atrial septum. And if the patients developed postcapillary pulmonary hypertension and did not really tolerate the situation with a restrictive atrial septum, we would see it in the intensive care unit. Then we 
would proceed; probably our cardiologist, would perform a percutaneous procedure to enlarge the atrial septum. But I cannot tell you numbers with regard to this study what it could be the clear indication to say we would do or not. In this group, that was not the case.

Dr Caldarone. Well, I can commiserate with you because when we look at our records and try to identify the subset of patients in whom a 2-ventricle repair was considered, it is hard to discern. So if we are going to really evaluate the use of hybrid management for 1-ventricle/2-ventricle decision making, as a community we are really going to have to identify those patients up front and say they are in this track and then monitor our outcomes to see how successful or unsuccessful we are. I think that is the only way we are ever going to really be able to assess this niche use of hybrid technology.

Dr Yerebakan. I absolutely agree with your comments and the scientific way to do it. My data also have the drawbacks of a retrospective design, so I am not really able to give clear data for your question.

Dr Sabine Daebritz (Duisburg, Germany). It is a very interesting approach. However, what is the rationale of doing this, particularly in the real hypoplastic left heart complex patients. What do you really gain? The decision is anyway very difficult, and there are no hard data to know whether a biventricular repair is going to work or not. The hypothesis is that it is probably most likely working in the neonate because there is some potential of even growth of the heart right after birth. So what do you really gain by adding this step in between?

And the other thing is, often you do not have a restrictive atrial septal defect, so how do you think you really promote growth of the left-sided structures, like in a very unbalanced atrioventricular septal defect or in a nonrestrictive atrial septal defect, if you do not have an intracardiac connection at the ventricular level, a ventricular septal defect.
Dr Yerebakan. So with regard to the second question maybe at first. I do not advocate that we really promote growth. I mean therefore to say we have to have hard data on this.

But what we see is when we apply the new discriminant score that was published in 2006 by Colan et al-this is with regard to your first question-what we gain with this approach is, you see, patients pretty much, as we expected, lie at the discriminant value of minus 0.65 . So 18 patients would only be amenable for biventricular correction if we would opt for a decision in the neonatal period. In contrast, when we go to the time of the biventricular correction, this score goes up to 1.47 , which means that the number of patients doubles. So we then have growth of the left ventricle structures, as we see with the score, and are sure, or let us say more sure, which patients would tolerate a biventricular correction.

Another reason why we choose this approach is there are data in the literature that tells us that in patients with complex heart disease, the brain maturation is delayed.

And second, the effects of a cardiopulmonary bypass and even hypothermic circulatory arrest in the newborn period has detrimental effects of the neurologic outcome. And what we have just-I am wrapping it up-just to show you, short results of the neurologic outcome; these are preliminary data. This is a study from the literature where we see that $40 \%$ of patients lie below the plus directional indicator of 70 . In our patients, just preliminary, unpublished data, in 19 patients in comparison to a hypoplastic left heart syndrome patients with conventional repair, only 5\% of our patients lie below the discriminate score of 70. And also with the minus directional indicator, this is the same case. So we have avoided neonatal major surgery, with cardiopulmonary bypass and we have at least seen in the preliminary data a satisfying neurologic outcome with this approach. But, as I said, this is just an alternative and only an institutional preference; nobody has to do it.

\title{
EDITORIAL COMMENTARY
}

\section{Kicking the can down the road ... gently}

\author{
Robert D. B. Jaquiss, MD
}

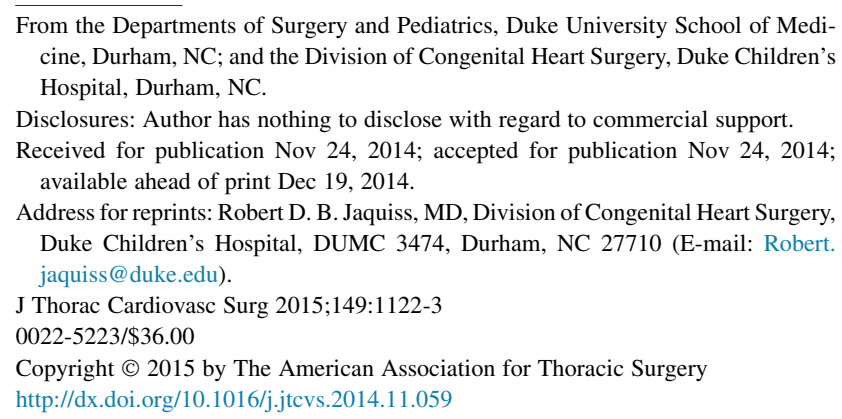

See related article on pages 1112-22.

Hypoplasia of left heart structures presents along a spectrum, ranging from the most severe, mitral and aortic atresia, to the least severe, coarctation of the aorta with bicuspid aortic valve. Two alternative treatment strategies now exist, with assignment of a patient either to a biventricular circulatory arrangement or to a univentricular arrangement, depending the perceived functional adequacy of the left side of the heart. Thus a "continuous" disease must be 


\section{APPENDIX E1. DETAILED DESCRIPTION OF CASES WHO DID NOT SURVIVE BIVENTRICULAR CORRECTION}

Two of the patients had syndromic features, 1 hypotrophic newborn infant (birth weight $=2.5 \mathrm{~kg}$ ) with a diagnosis of HLHC, and dysplastic aortic and pulmonary valves, had Robinow syndrome and died 4 weeks after direct biventricular correction of unknown reason. Another newborn infant (birth weight: $2.3 \mathrm{~kg}$ ) had microcephaly and dissymmetry of the head with a cardiac diagnosis of severe valvar and subvalvar aortic stenosis and hypoplastic aortic arch. He was waiting for the performance of a Ross-Konno procedure due to restenosis of the aortic valve after direct biventricular repair following failed interventional aortic valve dilation attempt. He died because of a progressive dilation of the left ventricle 4 months postoperatively. One patient with double-outlet right ventricle (Taussig-Bing anomaly) and hypoplastic aortic arch/aortic valve has been treated with arterial switch operation and reconstruction of the ascending aorta and aortic arch. She required postoperative venoarterial (3 weeks) and subsequent venovenous (3 weeks) extracorporeal oxygenation therapy (ECMO) and died as a result of a progressive right ventricular failure (severe pulmonary hypertension), cerebral infarction, and progredient liver failure during the second ECMO therapy 6 weeks postoperatively. A patient with HLHC, hypoplastic aortic arch, and endocardial fibroelastosis had to be converted to univentricular circulation using a Damus-Kaye-Stansel anastomosis and systemic to pulmonary shunt implantation after an attempt of direct biventricular correction. Postoperatively, he required 1 week of ECMO support but died as a result of several episodes of bacterial pneumonia, liver failure with severe coagulation derangement, and shunt thrombosis 5 weeks postoperatively. 
TABLE E1. Characteristics of patients who died after biventricular correction after hybrid stage I

\begin{tabular}{|c|c|c|c|c|c|c|}
\hline Diagnosis & Syndrome & BW (kg) & AV Z score & MV Z score & LV length $Z$ score & Reason of death \\
\hline HLHC, hypAoA, VSD & Robinow syndrome & 2.53 & -0.79 & 0.49 & -0.26 & Unknown \\
\hline AS, hypAoA, VSD & Unknown syndrome & 2.3 & -3.75 & 0.94 & -1.29 & LV failure \\
\hline $\begin{array}{l}\text { DORV, hypAoA, coronary } \\
\text { anomaly }\end{array}$ & - & 3.5 & -0.61 & 0.46 & -1.43 & $\begin{array}{l}\text { RV failure, liver failure, cerebral } \\
\text { infarction on ECMO }\end{array}$ \\
\hline HLHC, hypAoA, EFE & - & 3.3 & -2.13 & 0.48 & -3.28 & $\begin{array}{l}\text { Shunt thrombosis, liver failure } \\
\text { under ECMO after conversion } \\
\text { to UVC }\end{array}$ \\
\hline
\end{tabular}

$B W$, Birth weight; $A V$, Aortic valve; $M V$, mitral valve; $L V$, left ventricle; $H L H C$, hypoplastic left heart complex; hypAoA, hypoplastic aortic arch; $V S D$, ventricular septal defect; $A S$, aortic stenosis; $D O R V$, double-outlet right ventricle; $R V$, right ventricle; $E C M O$, extracorporeal oxygenation therapy; $E F E$, endocardial fibroelastosis; $U V C$, univentricular circulation. 